\title{
Poder territorial y recursos estatales: el Partido Autonomista Nacional durante la formación del Estado argentino, 1862-1916
}

Territorial power and state resources: the National Autonomist Party during the formation of the Argentinian state, 1862-1916

Jonás Chaia De Bellis ${ }^{*}$

Recibido el 5 de agosto de 2015 Aceptado el 5 de abril de 2016

Resumen: en este trabajo se analizaron las características organizacionales del Partido Autonomista Nacional argentino, entre 1862 y 1916, con atención especial en la competencia entre sus facciones territoriales y en el uso político de los recursos estatales. El periodo se reconstruyó desde un abordaje histórico-político, y se encontró que el resultado de la competencia entre facciones fue el desarrollo de instituciones estatales de alcance nacional, pero que al mismo tiempo, el empleo de esos recursos para la obtención de lealtad y adhesión de las facciones locales causó que la institucionalización del partido fuera débil. Discutiendo con las principales teorías sobre partidos políticos, un aporte de este trabajo es la conceptualización del partido como un "partido estatal de facciones".

* Consejo Nacional de Investigaciones Científicas y Técnicas. Instituto de Investigaciones Gino Germani, Universidad de Buenos Aires. Pte. J. E. Uriburu 950, 6to piso (C1114AAD), Ciudad Autónoma de Buenos Aires, Argentina. Correo electrónico: jonas.heliogabalo@gmail.com 
Palabras clave: Partido Autonomista Nacional argentino; partidos políticos; institucionalización de los partidos políticos; recursos estatales; formación del Estado argentino.

Abstract: in this paper the organizational characteristics of the Argentinian National Autonomist Party, between 1862 and 1916, were analyzed, with the main focus on the rivalry between its territorial factions and on the political use of state resources. This period was reconstructed from a historical-political approach, and it was found that the result of the rivalry between factions was the development of national-level state institutions, but, at the same time, the use of these resources to win allegiance and adherence from local factions led to a weak institutionalization of the party. After discussing the main theories on political parties, a contribution of this paper is the conceptualization of the party as a "factional state party".

Key words: Argentinian National Autonomist Party; political parties; institutionalization of political parties; state resources; formation of the Argentinian state.

\section{Introducción}

En este trabajo se analizan algunas características organizacionales del Partido Autonomista Nacional (PAN), con atención especial en la competencia entre sus facciones territoriales y en el uso coalicional de los recursos estatales. La hipótesis general es que el resultado de la competencia entre las facciones fue la homogeneización del Estado y el desarrollo de instituciones estatales de alcance nacional, pero que dichos recursos -empleados para obtener la lealtad y adhesión de las facciones territoriales- causaron, al mismo tiempo, que la institucionalización del PAN fuese débil. Otras hipótesis que se sostienen aquí son: a) el PAN manifiesta características de faccionalismo degenerati- 
vo, en tanto que es posible observar fragmentación excesiva, incentivos "privatizantes", y de faccionalismo enraizado; b) además de los recursos estatales, la posibilidad de colonizar las burocracias y la baja competencia electoral incidieron en su institucionalización; c) el PAN fue un partido orientado a los cargos maximizador de beneficios materiales de los líderes de cada facción; d) la categoría de "partido de notables" no es aplicable del todo al PAN, debido a las características del ciclo institucional y de la competencia oligopólica entre facciones, que más bien presenta rasgos de cartelización o propios del "Estado de partidos". Por tanto, a partir de estas hipótesis, aquí se propone el concepto de "partido estatal de facciones" para categorizar al PAN.

El trabajo se dividió en tres partes; en la primera se reconstruyeron históricamente los procesos de formación del Estado argentino (desde 1862) y del gobierno presidencial del PAN (1874-1916); se prestó atención tanto a los recursos estatales, empleados para el armado de coaliciones, como a la competencia entre las facciones. En la segunda parte se revisaron teorías sobre los partidos políticos utilizadas en la tercera, para explicar el proceso de institucionalización del PAN.

\section{Recursos estatales y competencia política: el PAN y el proceso formativo del Estado argentino}

En este apartado se hizo una reconstrucción breve de los procesos paralelos y conexos de formación del Estado nacional (1862-1916) y de competencia entre facciones político-territoriales, ${ }^{1}$ en los que el PAN fue un actor crucial. Tanto en el periodo que siguió a la independencia argentina de la Corona española, como en el que se revisó aquí, hubo conflictos alrededor de la "posesión de los derechos adua-

1 Aquí se definirá a las facciones como las subdivisiones de un partido político en grupos fuertemente organizados nacionalmente, de modo que la coalición dominante del partido se encuentra débilmente cohesionada; así, los intercambios políticos entre elites partidarias y seguidores son realizados por una pluralidad de líderes. Las facciones se distinguen analíticamente de las tendencias puesto que las segundas se producen en el vértice de la organización partidaria y carecen de apoyos fuertes o bases organizadas, mientras que las facciones, por el contrario, atraviesan al partido desde el vértice hasta las bases, constituyendo así facciones nacionales (Panebianco 1993, 92-93). 
neros", en tanto que "ingresos casi excluyentes del Estado" (González 2000, 120).

En efecto, luego de la conformación de la primera autoridad política autónoma, en 1810, y de la independencia definitiva respecto de la metrópoli, en 1816, el mantenimiento de la unidad territorial del exvirreinato del Río de la Plata se volvió una cuestión crucial para cualquier tentativa de gobierno nacional; desde mediados de 1820 , cuando comenzaron a enfrentarse en una guerra civil prolongada que duraría hasta 1880 , dos tendencias políticas con proyectos opuestos de organización territorial: el unitarismo, que buscaba "heredar el poder político del gobierno imperial”; a partir de la hegemonía aduanera portuaria de la de Buenos Aires, y el federalismo que defendía la soberanía política de las entidades provinciales autónomas, buscaba la distribución de la renta aduanera controlada por Buenos Aires, y también la conexión y recuperación de los circuitos productivos del interior mediterráneo y litoral del país, estancados tras la disolución virreinal (Oszlak 2009, 47 y 51; Ternavasio 2009, 119).

Pese a estas tendencias en guerra, las fuerzas federales no se concentraron sólo en el interior del país, ni las fuerzas unitarias lo hicieron en Buenos Aires, sino que en todo el proceso hubo alianzas cruzadas de liderazgos interprovinciales, por lo que el acceso a la presidencia de líderes del interior no condujo al cese de la guerra civil, ni las presidencias de líderes porteños implicaron la ausencia de apoyos provinciales para ellos (Bragoni y Míguez 2010, 24-25).

Para analizar el proceso de formación estatal argentino, Rock y López-Alves (2000) se concentraron en la consolidación del poder y la autoridad central en los gobiernos de Bartolomé Mitre, Nicolás Avellaneda y Julio Argentino Roca, el primero líder del Partido Liberal y los segundos del PAN; ésta se llevó a cabo a partir de la guerra civil, la coerción y la creación de vínculos políticos interregionales basados en el patronazgo y los subsidios. Los autores afirman que la clase terrateniente argentina fue más poderosa que la comercial, y su alianza con el Ejército pudo dar forma a un Estado capaz de garantizar las condiciones para un desarrollo económico rápido; así, cuanto más se fortalecía el Estado, la competencia político-partidaria se hacía más débil, por lo que la formación de un "bloque conservador" controlado por los terratenientes contribuyó al desarrollo económico y a 
preservar el dominio político de la oligarquía terrateniente (Rock y López-Alves 2000, 176-178 y 180-181).

Los autores dividen el proceso de formación estatal en dos fases: a) la del Partido Liberal, liderado por el presidente Mitre y b) la del PAN, encabezado por los presidentes Avellaneda y Roca. Durante la primera fase (1860), la estructura del Estado fue débil, ya que carecía de capacidades para controlar los poderes provinciales; la consecuencia fue que Buenos Aires adquiriese un poder predominante. Durante la segunda $(1874,1880)$, las provincias revirtieron esta situación y así lograron influir, participar y dar forma al Estado (Rock y López-Alves 2000, 179).

Rock y López-Alves $(2000,186)$ señalan que la primera fase comenzó tras la caída de Juan Manuel de Rosas, en 1852, pero debido a la resistencia de Buenos Aires a subsidiar al resto de las provincias, el resultado fue un Estado precario, denominado Confederación Argentina, conformado por todas, excepto la de Buenos Aires. Cuya prosperidad, como Estado independiente, contrastaba con la bancarrota de la Confederación, ya que la provincia autónoma contaba con un cuasi monopolio del comercio exterior. Hacia 1859, el presidente de la Confederación, Justo José de Urquiza, invadió Buenos Aires para lograr su cooperación económica, y que pasara a formar parte de ella. Los enormes e insostenibles gastos militares, empleados para la defensa de la provincia, llevaron a que Dalmacio Vélez Sarsfield del partido mitrista- propusiese un esquema para trasladar el control provincial de los ingresos aduaneros y los gastos militares a la nación, a cambio de que el Estado devolviera una compensación en subsidios igual o mayor a los gastos corrientes; pero cuando Buenos Aires incrementó su apoyo a las fuerzas mitristas distribuidas en las provincias, el acuerdo cayó y de nuevo se desató una guerra que ganó Buenos Aires. En 1862 Mitre se convirtió en presidente de una república que incorporaba a todas las provincias.

Durante la presidencia de Mitre el Partido Liberal se partió en dos: los autonomistas -la facción liderada por Adolfo Alsina-, que buscaban preservar el poder autónomo de Buenos Aires, y los nacionalistas -la que respondía a Mitre-, que querían establecer un gobierno nacional central, sin abandonar el predominio porteño (Rock y LópezAlves 2000, 186). 
El resultado de la presidencia de Mitre en cuanto al desarrollo de un Estado central fue distinto al alcanzado en la provincia de Buenos Aires, en tanto que estado provincial, ya que Mitre fue incapaz de imponer su autoridad en las provincias restantes, sobre todo en el noroeste, que afrontaba una situación económica crítica que desató rebeliones populares y conflictos armados; Mitre, tras derrotar a los caudillos, colocaba gobernadores afines quienes carecían del apoyo de la población local. Al mismo tiempo, la guerra de la triple alianza contra Paraguay también desató rebeliones, ya que la población de las provincias se resistía al reclutamiento forzoso. No obstante, los resultados de la guerra fueron favorables para la provincia de Buenos Aires, que repercutieron también en el desarrollo de las instituciones estatales: las inversiones de Brasil (aliado en la guerra) y el comercio con las provincias del litoral incrementaron la recaudación del Estado provincial y le proveyeron los recursos necesarios para controlar a las rebeldes, y así el Estado nacional se trasformó en uno "predatorio" (Rock y López-Alves 2000, 187-188). ${ }^{2}$ Sin embargo, el mecanismo para la obtención de adhesiones nacionales por parte de Mitre fue el Ejército o la "penetración estatal represiva", ya que el conflicto faccioso y la imposibilidad estatal de mantener una presencia institucional permanente, capaz de anticipar y disolver las oposiciones en nombre del Estado nacional, frustró la creación de instituciones de alcance nacional (Oszlak 1982, 9-10). De hecho, una coalición entre la facción de Alsina y el general Arredondo, un militar que controlaba varias provincias, ofreció su apoyo a Domingo Faustino Sarmiento, en 1868, y a Avellaneda, en 1874, quienes alcanzaron la presidencia nacional.

La coalición presidencial de Avellaneda estaba conformada por gobernadores de una clase naciente de terratenientes provinciales, asociada al crecimiento del comercio y la producción agraria, azucarera, vitivinícola y algodonera, que para apoyar a uno de sus miembros formó la Liga de Gobernadores, cuyo rol crucial en el triunfo de Avellaneda demostraba que una de las claves para la supremacía política nacional dependía de la capacidad de obtener el apoyo de las provin-

2 Oszlak (1982, 7-8) señala que el empleo de recursos y organismos provinciales por parte del Estado mitrista implicó una ampliación de la actividad estatal que luego se perfeccionaría y consolidaría con el gobierno de Roca: el monopolio de la emisión monetaria, la construcción de un ejército, de un aparato recaudatorio y de un sistema bancario nacional. 
cias. Aunque esta liga se apoyaba sobre sectores del interior, Alsina fue igualmente recompensado con los cargos de vicepresidente de Sarmiento y ministro de Guerra de Avellaneda. En 1874, esta alianza de gobernadores provinciales y Buenos Aires, con representación presidencial, se denominó Partido Autonomista Nacional (Rock y LópezAlves 2000, 191-192). Desde que la coalición autonomista ocupó la presidencia, esto es, la segunda fase de formación del Estado, el desarrollo de las instituciones estatales para el control de las provincias se relacionó con los vínculos de patronazgo que complementaron a los coercitivos; los subsidios del Estado central a las provincias, por ejemplo los provistos por el nuevo sistema educativo, impulsado por Sarmiento y Avellaneda -su ministro de Educación-, fueron recursos decisivos para acceder al poder. Por otra parte, el cargo de gobernador era la otra clave del sistema de poder nacional, ya que éste no sólo podía intercambiar su apoyo político al presidente por redes ferroviarias u obras públicas, sino que la propia administración provincial era proveedora de empleo (designaciones a familiares y seguidores como personal administrativo o escolar). No obstante, los gobernadores necesitaban a su vez el respaldo de los militares del nuevo ejército autonomista, distribuidos por todo el país, y de los jueces de paz, quienes movilizaban los recursos para la legitimación electoral de los candidatos (Rock y López-Alves 2000, 191-192).

En la elección de Roca como presidente también se observa la necesidad y efectividad del apoyo de las facciones locales. En 1878, Carlos Tejedor, gobernador de Buenos Aires, quien contaba con el respaldo de algunas facciones provinciales, manifestó su voluntad de ser presidente; la Liga de Gobernadores volvió a funcionar como núcleo del PAN, y ofreció su cooperación a Roca, ${ }^{3}$ el ministro de Guerra de Avellaneda, lo que éste pagó con las tierras adquiridas durante la

3 La trayectoria de Roca revela mucho acerca de la relación entre facciones partidarias, Estado y poder económico. Como líder militar, Roca había construido una base importante de apoyo en el país e incluso en Buenos Aires, tras haber derrotado los levantamientos de los caudillos provinciales y liderado la campaña al desierto. Al mismo tiempo, Roca contrajo matrimonio con una mujer de la clase terrateniente de Córdoba, y pronto se convirtió en administrador de tierras. Cuando sucedió a Alsina, en el Ministerio de Guerra, formó milicias provinciales con armas compradas a Estados Unidos para enfrentar el levantamiento porteño liderado por Carlos Tejedor, cuya derrota, en 1880, tuvo como resultado la Ley de Federalización (Rock y López-Alves 2000, 194). 
campaña al desierto. En efecto, Adamovsky (2009) señala que la Liga de Gobernadores supuso para su coordinación una serie de acuerdos entre grupos de poder provincial, basados en beneficios asociados al desarrollo de la economía agroexportadora. Tanto la campaña al desierto como la incorporación de otras tierras sin titular legal de la región pampeana y del Chaco formaron parte de un proceso inmenso de privatización de tierras organizado por el Estado. ${ }^{4}$ Pero el resultado del modo en que éstas se privatizaron y las características del mercado inmobiliario fue que se concentraron en pocos terratenientes (Adamovsky 2009, 33). Es interesante observar cómo el régimen político cristalizaba la dinámica de intercambios y dependencias entre los poderes nacionales y los locales. Botana $(2012,64)$ señala que el régimen oligárquico funcionó como un sistema de "hegemonía gubernamental" a partir de la "unificación del origen electoral" de los cargos de gobierno. La capacidad del gobierno nacional para nombrar sucesores y el control sobre los gobiernos provinciales dependían menos de las elecciones populares que de la decisión del presidente, ya que él era el alfa y el omega del sistema. En efecto, él seleccionaba al gobernador y ambos, de común acuerdo, elegían diputados y senadores, quienes luego decidían acerca de la composición de las legislaturas provinciales (Botana 2012, 64). Otro aspecto importante es que los gobernadores también confeccionaban la lista de electores del presidente (las juntas de electores). Así "se explica el intercambio recíproco entre Nación y Provincias, porque sin el apoyo de los gobernadores el poder presidencial carecía de sustento, pero sin el resguardo nacional los gobernadores permanecían huérfanos de la autoridad indispensable para mandar en su ámbito particular" (Botana 2012, 88).

Como sostienen Rock y López-Alves (2000, 200), si “durante el período del dominio de Roca el Estado se transformó casi en un sinónimo del PAN dirigente", es interesante describir, en forma muy breve, el funcionamiento que adoptó ese Estado, ya que los recursos estatales enumerados hasta aquí (control militar, subsidios, empleo público, obras públicas, privatizaciones y redes ferroviarias) influyeron en el tipo de institucionalización partidaria. Además de la "penetración re-

4 Así, en 1903, Roca podía afirmar que, hasta ese momento, se habían concedido a manos privadas más de 32 millones de hectáreas (Adamovsky 2009, 33). 
presiva" mencionada, a partir de los gobiernos del PAN se pusieron en práctica otros patrones de actividad estatal, tales como la "penetración material" o "cooptativa"5 del Estado (Oszlak 1982, 10).

Este tipo de acción se orientó a regular los factores de producción nacionales mediante la provisión de recursos técnicos, financieros y legales (códigos) para el desarrollo de obras de infraestructura y el suministro de servicios; el Estado patrocinaba negocios ofreciendo beneficios, garantías y privilegios a empresarios privados, tales como la construcción de servicios y redes ferroviarias que valorizaban tierras y propiedades al tiempo que dinamizaban y unificaban los circuitos productivos (Oszlak 1982, 10 y 15). Señala Oszlak que

mediante la cooptación, el estado nacional intentó ganar aliados entre fracciones burguesas del interior y gobiernos provinciales, a través de la promesa cierta o efectiva concesión de diversos tipos de beneficios conducentes a incorporar nuevos grupos o sectores a la coalición dominante. Por ejemplo, el selectivo empleo de subsidios, ${ }^{6}$ el nepotismo en la designación de cargos públicos nacionales, o la intervención federal dirigida a instalar o reponer en el gobierno de las provincias a aliados de causa de los sectores que ejercían el control del gobierno nacional, fueron algunos de los mecanismos de cooptación empleados $(1982,10)$.

5 En un reciente e innovador estudio, Bragoni y Míguez $(2010,27)$ afirman que la imagen planteada por Oszlak acerca de una "penetración" de la periferia por parte de un poder central, en tanto que dinámica principal de la formación del Estado argentino, podría ser matizada a partir de la consideración de las situaciones e intereses provinciales. Los autores señalan que durante el período revisado aquí, el Estado central se conformó mediante la "integración de las situaciones provinciales": "la presencia de la Nación en las provincias [...] no aparece como la penetración de un actor ajeno que las va conquistando o sometiendo [...] sino más bien como la construcción de un conjunto de acuerdos y de instituciones que las propias elites provinciales establecieron sobre la base de un ejercicio político empírico de ensayo y de error, en el cual prevalecieron intereses de naturaleza variada, aunque sujetos a instrumentos y mecanismos simultáneos implementados por los poderes locales como por los representantes o comisionados del poder central".

6 Un caso interesante de subsidios es el de las "colonias oficiales": la colonización de territorios por parte de inmigrantes era sostenida por el gobierno central mediante la distribución de subsidios a las provincias receptoras, que se beneficiaban al contar con más tierras destinadas a la producción, con nuevos propietarios y con más fuerza de trabajo (Oszlak 2009, 148). 
Por ejemplo, las negociaciones previas y posteriores a las elecciones provinciales implicaban remuneraciones políticas de los acuerdos mediante cargos en la administración pública, lo que llevaba a un crecimiento rápido del empleo público local; en 1876, de 12835 empleados, 10956 se desenvolvían en las administraciones provinciales (Oszlak 2009, 126). El ferrocarril fue otro recurso estatal para obtener la lealtad coalicional de las facciones territoriales. En primer lugar, la discrecionalidad tarifaria del gobierno central sirvió "como un instrumento clave para favorecer o perjudicar el desarrollo de las diferentes regiones del país", benefició a los terratenientes de la Pampa húmeda y del Litoral, y marginó a la región cuyana y a las provincias de Salta o Santiago del Estero (Oszlak 2009, 146-147). La llegada de la empresa pública Ferrocarril Central Norte, a mediados de 1870 a Tucumán, fue resultado de un acuerdo entre las elites locales productoras de azúcar que podrían reducir los costos de trasporte, imponer aranceles proteccionistas y ubicar su producción en Buenos Aires y el gobierno central, que lograría un desplazamiento de tropas rápido y eficiente, para profundizar las redes clientelares en el interior del país (Sánchez Román 2010, 223-224); ; el ferrocarril, además, fue el paso siguiente a la llegada de las sucursales de los bancos estatales a la provincia, como el Banco Nacional, principal fuente de financiamiento de las elites azucareras desde 1875 (Sánchez Román $2010,221)$. Es posible observar otros procesos de intercambio entre recursos estatales y lealtad política en Tucumán, como la distribución de cargos en el gabinete del Poder Ejecutivo nacional. Por ejemplo, Herrera $(2010,206)$ señala que con estos cargos se recompensaba a las facciones locales del PAN, encargadas de neutralizar las rebeliones provinciales opositoras al gobierno nacional durante la presidencia de Sarmiento (1868-1874); así, Uladislao Frías, líder del PAN tucumano, gobernador provincial, y encargado de fomentar la alianza entre las elites azucareras y el gobierno central, se convirtió en ministro del Interior, de Sarmiento, y desde ese cargo él y otros integrantes de la elite económica tucumana promovieron el desarrollo de sus ingenios azucareros. Las agencias judiciales nacionales se usaron como inter-

7 Otro beneficio derivado de los ferrocarriles que recibieron las provincias fueron los empleos; en 1883, la construcción de diez ferrocarriles involucraba 14500 puestos de trabajo (Oszlak 2009, 149). 
cambio político, asociado al desarrollo del Estado nacional. Por ejemplo, Paz (2010, 161-166) describe las primeras interacciones entre las facciones del PAN, adeptas al presidente Avellaneda en la provincia de Jujuy y la nueva Corte Suprema de Justicia de la Nación, y analiza una controversia suscitada en torno a los derechos de propiedad de amplios territorios de la puna jujeña. La Corte Suprema otorgó al fisco provincial esas tierras contra el reclamo de propiedad hereditaria de la familia Campero vinculada a la facción mitrista del PAN, y de este modo le garantizó a la provincia tanto la posibilidad de controlar coercitiva e impositivamente a la población rural de campesinos indígenas, que se habían rebelado en numerosas oportunidades contra la autoridad provincial, como de obtener beneficios de la venta o de los arriendos de esas tierras.

Si bien es posible observar un "bloque conservador", ni la dinámica del régimen político y tampoco las prácticas estatales de cooptación deben ofrecer la imagen de un sistema homogéneo y cooperativo de intercambios y apoyos; en realidad, durante los años del PAN es posible observar un alto grado de faccionalismo dentro y fuera del autonomismo. El "mapa de grupos políticos", elaborado por Botana (2012, 274-275), puede ser muy útil para observar el juego de facciones durante esos años. Entre 1854 y 1910 existieron cerca de 23 grupos políticos, conformados a partir de desprendimientos de líderes que abandonaban (y en algunos casos retornaban) a sus facciones de origen, nueve de los cuales se produjeron entre 1874 y 1890 ; por otra parte, el PAN tuvo diez rupturas, entre 1874 y 1910 , lo que no impidió que una de sus facciones siempre ocupase la presidencia nacional durante todo el periodo, de 1874 a 1916. En este sentido, Alonso señala que "la ausencia de competencia entre partidos políticos antagónicos fue reemplazada en esos años por la que se originó rápidamente dentro de las filas del partido único" $(2003,279)$.

Alonso contabiliza cuatro ligas dentro del PAN, entre 1881 y 1885 , sostiene que la principal era la liderada por el presidente Roca, quien contaba con recursos superiores a los de las ligas rivales para controlar el proceso político y mantener la supremacía de su facción leal; estos recursos eran distributivos/administrativos (empleos y obras públicas), distributivos/militares (armamentos) e institucionales/coercitivos (intervenciones federales). Para Alonso, la segunda liga más im- 
portante era la de Dardo Rocha, gobernador de la provincia de Buenos Aires, quien contaba con el Banco de la Provincia de Buenos Aires, lo que le brindaba autonomía y recursos financieros para conformar apoyos propios. Rocha abandonó a Roca una vez que llegó a la gobernación provincial, y comenzó su carrera hacia el poder nacional, lo que significaba para la liga roquista una amenaza hacia su proyecto de Estado nacional. Luego, señala Alonso, seguía la liga de Miguel Ángel Juárez Celman, gobernador de Córdoba, entre 1880 y 1883 , y senador, entre 1883 y 1885. Juárez Celman construyó una base de poder importante en su provincia y adquirió influencia en otras, pues su propósito también era ser presidente; no obstante, a diferencia de Rocha, nunca se confrontó abiertamente con Roca, aun cuando hasta 1885 no contó con la garantía de suceder al presidente. En una situación similar de incertidumbre se encontraba el líder de la cuarta facción, Bernardo de Irigoyen, ministro de Relaciones Exteriores y del Interior de Roca; si bien era la liga menos poderosa, su liderazgo podía quebrar los acuerdos provinciales en caso de abandonar al presidente y aliarse con facciones opositoras (Alonso 2003, 281-283).

Además de los recursos estatales mencionados, es interesante observar cómo se resolvieron las adhesiones y los conflictos políticos en las provincias durante la presidencia de Roca (1880-1886); Alonso (2003, 285-289), describe detalladamente este proceso en cada una. Según la autora, durante el primer gobierno de Roca, las de San Luis y de Mendoza se mantuvieron bajo la égida presidencial, y Buenos Aires en la oposición, a pesar de los intentos roquistas de organizar una facción leal contra Rocha. En otras provincias, el presidente se encargó de controlar a las facciones mediante acuerdos que garantizasen a los sectores leales una posición de poder. En La Rioja, donde una facción respondía a Juárez Celman y otra al presidente, Roca logró que cada una se alternase en el gobierno provincial siempre y cuando ambas respondiesen a sus órdenes en cuanto a la sucesión presidencial y a los cargos legislativos y ministeriales. En Jujuy, Roca aglutinó facciones locales para impedir que los sectores leales al gobernador porteño le arrebatasen el gobierno provincial, y logró también su alternancia en éste. En Córdoba, una facción respondía a Juárez Celman y otra a Roca; si bien Roca logró imponer a Ambrosio Olmos, éste sería destituido por un juicio político activado por la facción juarista. En la 
provincia de Santa Fe, si bien De Irigoyen contaba con ciertos sectores que lo apoyaban, como el hermano del caudillo provincial Simón de Iriondo, Agustín de Iriondo y el juarismo tenía presencia en el sur, Roca logró imponer a José Gálvez, ministro de gobierno, como gobernador, a cambio de que en las elecciones presidenciales apoyara a Juárez Celman.

En otras provincias, señala Alonso, la competencia entre facciones se resolvió mediante la competencia electoral: San Luis, en 1884 (entre las de Rocha y Roca para gobernador); Santiago del Estero, en 1882 (entre las de Rocha, Roca y Juárez Celman); entre Ríos (entre la de Rocha por un lado, enfrentada a una alianza entre las de Roca y Juárez Celman, por el cargo de gobernador); Tucumán, en 1886 (entre la de Irigoyen y Juárez Celman por las elecciones presidenciales) y Salta, en 1886 (todas las facciones compitieron en las elecciones presidenciales). En otras provincias, los conflictos entre facciones se resolvieron mediante la intervención federal y las revoluciones: en Santiago del Estero (intervención federal de Roca, en 1882, para imponer al juarismo, y revolución de la facción roquista en 1885, para controlar la provincia); en Catamarca (intervención federal de Roca, en 1884, para arrebatarle el control a la facción rochista); en Corrientes (revolución roquista liderada por Manuel Derqui, en 1882, y elección posterior de éste para gobernador en 1883) y en San Juan (asesinato de Agustín Gómez, en 1884, aliado a Rocha) (Alonso 2003, 285-289).

Como se mencionó, Roca había desarrollado una variedad de formas de controlar los procesos políticos provinciales; pero Juárez Celman, el sucesor presidencial "designado" en 1886, llegaba al poder sin apoyos propios, por lo que su estrategia para mantenerse fue distinta: no entrometerse en la política provincial y ofrecer autonomía económica; en efecto, si Roca, además de los mecanismos descritos, había intentado obtener la lealtad de las facciones provinciales mediante el acceso de las provincias al crédito desde el Banco Nacional y las obras públicas, Juárez Celman les ofreció libertad crediticia, a través de la Ley de Bancos Garantidos y acceso a las obras públicas, mediante la expansión del sistema ferroviario, decidida por el Congreso Nacional y no por el Ministerio del Interior, como en el caso de Roca (Alonso 2009, 376-377). 
Cuando Juárez Celman comenzó su mandato sólo contaba con el control de Córdoba (la policía, la legislatura y los ministros), del ministro de Guerra (Eduardo Racedo), además del apoyo de algunas facciones en Capital Federal, Tucumán y Santiago del Estero; el resto de los ministros nacionales eran leales a Roca, y las provincias se dividían entre las ligas mencionadas. Sin embargo, como resultado de la estrategia de autonomía política y económica para los líderes provinciales, Juárez Celman obtuvo el apoyo de las facciones, los gobernadores de Salta, Corrientes, Catamarca, San Juan y Santa Fe reemplazaron a los roquistas de los puestos administrativos y políticos, y ofrecieron su apoyo al presidente (Alonso 2009, 374-379). No obstante, Juárez Celman abandonó el poder de forma anticipada, en 1890, debido a una combinación de factores, entre ellos los efectos de la crisis económica y su competencia con Roca por el control de las facciones provinciales mencionada arriba. Pero la "revolución del parque" ese mismo año, llevada a cabo por la Unión Cívica (liderada por Mitre, De Irigoyen y Leandro Alem), fue un factor determinante.

La Unión Cívica también sufrió fracturas partidarias, y una de sus facciones se trasformó en una organización más estable: la Unión Cívica Radical (UCR), liderada al principio por De Irigoyen y Alem, y luego por Hipólito Yrigoyen. Si bien aquí se analizan algunas características del PAN, es interesante mencionar que en cuanto a la movilización electoral, a los incentivos distribuidos por los líderes partidarios y a las redes locales, la UCR desarrolló en sus inicios mecanismos políticos similares a los del PAN. En este sentido, Roy Hora (2001, 54) señala que la particularidad del radicalismo no pareció residir en la expresión política de los cambios socioeconómicos ni en la incorporación de los nuevos actores sociopolíticos surgidos de esos cambios, sino en el perfeccionamiento, la extensión y la coordinación del sistema de redes locales que también empleaba el PAN, y con el que la UCR obtuvo las victorias electorales de 1894 y 1895. En efecto, los "aparatos electorales" del PAN y del mitrismo eran similares a los de la UCR: las bases populares eran movilizadas por caudillos locales que respondían a las elites dirigentes, y la relación entre estos últimos no era espontánea sino que debía reconstruirse permanentemente mediante un sistema de intercambios materiales: "los líderes locales obtenían sus apoyos gracias a su propio ascendente personal, que en 
general se basaba en su capacidad para proveer un amplísimo conjunto de servicios, entre los que se cuenta [...] el empleo público" (Hora $2001,55){ }^{8}$

La movilización electoral durante los años del PAN y de la UCR, empleada para resolver conflictos entre las facciones, es un elemento que vuelve a cuestionar la impresión de que un "bloque conservador" manipulaba a un régimen político no competitivo. Si bien las elecciones no constituían el origen de los cargos políticos, su utilización como método resolutivo de conflictos y como proceso de legitimación de los líderes permite observar una función de los agrupamientos políticos de la época a la que dedicaron recursos importantes. Aquí ya se mencionó el sistema de movilización activado por gobernadores, caudillos locales, militares y jueces de paz, algunos estudios demuestran que éste no sería el único que explicara los apoyos obtenidos por cada facción en las elecciones, ya que es posible observar algunos patrones de continuidad en los sectores que ayudaron a cada partido en elecciones a lo largo del tiempo, es decir, una relación clase/voto. Cantón y Jorrat (2004) muestran que en las elecciones de la ciudad de Buenos Aires, entre 1864 y 1910, los sectores populares votaron por el PAN y los medio altos por el mitrismo. Los autores también revisan la competitividad de las elecciones en ese periodo, y muestran que la mayoría (19 sobre 29) fueron mediana y altamente competitivas, que tres tuvieron baja competitividad y que siete no fueron competitivas (Cantón y Jorrat 2004, 13). ${ }^{9}$ A partir de las pautas ocupacionales registradas, los obreros no calificados votaron por el PAN, y los profesionales, empresarios y comerciantes por los grupos liderados por Mitre. En este sentido, es posible afirmar que existió un voto de clase, aun si los partidos no expresaban políticas de clase (Cantón y Jorrat 2004, 24). Lo más interesante que proponen los autores es que la relación entre ocupación y voto no permite sostener que el comportamiento electoral del periodo haya sido aleatorio o producto de las maquinarias electorales. En efecto, señalan que si estas maquinarias "hubiesen

8 Para un análisis detallado de las "maquinarias electorales" del radicalismo véase Horowitz (2007), Rock (1972) y Walter (1974).

9 Las "elecciones sin competitividad" son en las que se presentó un partido, las de "baja competitividad", en las que el partido ganador obtuvo 80 por ciento o más de los votos, y las de "media" y "alta competitividad", en las que se presentó más de un partido, y el ganador obtuvo menos de 80 por ciento de los votos (Cantón y Jorrat 2004, 13). 
mostrado la eficiencia y amplitud que se les adjudica [...] sería difícil, por no decir imposible, detectar pautas consistentes de asociación ocupación-voto como las que se encuentran" (Cantón y Jorrat 2004, 28-29). Otro hecho interesante, relativo a los procesos electorales y a las facciones territoriales, es la "conciliación de los partidos", que tuvo lugar en 1877, también en Buenos Aires, analizada por Lettieri (1999).

Luego de la derrota militar del levantamiento de Mitre contra el PAN -a raíz de la victoria de Avellaneda como presidente en 1874-, la facción porteña mitrista decidió abstenerse de todas las elecciones y seguir sosteniendo la vía revolucionaria. La automarginación del mitrismo obligó a que el PAN cumpliera "simultáneamente los roles de oficialismo y oposición en el seno de las instituciones nacionales y provinciales" (Lettieri 1999, 35). En Buenos Aires, los líderes más jóvenes del PAN (Aristóbulo del Valle, Alem y Rocha) iniciaron una embestida contra los dirigentes más antiguos del autonomismo. El presidente Avellaneda afirmaba que "en la vida hay contrapesos indispensables, y cuando no los establece la separación franca del adversario, se los busca en las divisiones internas del mismo partido [...]" (Lettieri 1999, 43).

Pero estas divisiones amenazaban con incrementar el conflicto político: la provocación revolucionaria de Mitre, la crisis del Tesoro Nacional de 1873, los levantamientos del caudillo Ricardo López Jordán, la guerra de la triple alianza y el aplastamiento de las facciones opositoras en las provincias por parte del Ejército, entre otras cosas, demostraban que la fractura del PAN en Buenos Aires sería la gota que derramaría el vaso, de modo tal que Avellaneda intentó reincorporar institucionalmente al mitrismo para, al menos, reequilibrar la política provincial. El plan de reincorporación política de 1877 se denominó Conciliación, y consistió en un acuerdo entre Avellaneda y Carlos Casares (gobernador de Buenos Aires) según el cual el mitrismo volvería a competir en las elecciones y depondría las armas, y así evitaría la aparición de más facciones y conflictos armados (Lettieri 1999, 75-80). En síntesis, Alonso $(2009,370)$ señala que pertenecer al PAN significaba apoyar públicamente a un candidato presidencial, ya que una vez finalizadas las elecciones quedaban estructuras de poderes provinciales que sólo se vinculaban al gobierno nacional hasta la próxima elección. 
Vale la pena citar la descripción de Alonso sobre el PAN:

El partido no gozó de organización interna alguna, ni tampoco resolvió a través de acuerdos formales o informales cuáles serían los métodos de selección de candidatos a puestos electivos. El PAN consistió en un sistema informal de vinculación de distintos líderes provinciales y nacionales que decían pertenecer al partido. Dentro de este sistema, quienes aspiraban a la presidencia reclutaban apoyos en aquellas fuentes de poder electoral constituidas por quienes dominaban o decían dominar la política en sus provincias y podían producir los votos necesarios llegado el momento de la elección. Los acuerdos forjados formaban coaliciones, denominadas ligas. Además de la liga del presidente, que intentaba controlar la política nacional y la siguiente elección presidencial, había tantas ligas como candidatos con posibilidades de éxito de alcanzar la presidencia. La dinámica generada, por lo tanto, era la de ardua competencia entre las distintas coaliciones $(2009,371)$.

\section{Partidos políticos: liderazgos, competencia y Estado}

En este apartado se revisan algunos análisis sobre la organización interna de los partidos políticos y sus relaciones con el entorno, que serán retomados más adelante para analizar al PAN.

Definiciones de partido: relación con la competencia, el acceso al poder, el reclutamiento de elites y el liderazgo

Algunos análisis sobre los partidos políticos los definen considerando el entorno de competencia abierta en el que actúan. Según Katz y Mair $(2004,11)$, muchas de las definiciones sobre los partidos políticos se pensaron tomando a los partidos de masas y los requisitos institucionales para su existencia como modelo: los grupos que participan en la vida política estarían predefinidos, la política consistiría en la competencia, cooperación y conflicto entre ellos, y los partidos serían los agentes mediante los cuales estos grupos participan políti- 
camente, formulan demandas al Estado y lo intentan controlar con el acceso de sus candidatos a los cargos públicos.

Entre las definiciones que consideran a los partidos asociados a formas competitivas de democracia está la de Yanai (1999, 5-11), quien afirma que a pesar de su declive como organización y la pérdida de sus roles políticos y sociales, éstos sobreviven en todos los regímenes políticos que se legitiman mediante el consenso y la representación. Los partidos, al margen de sus trasformaciones constantes, desempeñan sus roles exclusivos institucionales sólo en un régimen democrático, que por lo regular administran dos procesos centrales: la organización de elecciones competitivas y la faccionalización de la legislatura y del gobierno. Para Ignazi (1996, 551-552), los partidos tendrían tres funciones: la estructuración del voto, la selección del personal político y la formulación de políticas, aunque sólo la primera es competencia exclusiva de ellos. Schlesinger (1991) sostiene que un partido político es un grupo organizado para ganar el control del gobierno en su nombre mediante el triunfo en elecciones para cargos públicos, mientras que Downs (1957) afirma que es una coalición de personas que busca el control del aparato gubernamental por medios legales. Epstein (1967) define a los partidos como cualquier grupo, sin importar que se organice en forma débil, que busque ser electo para ocupar puestos de gobierno bajo un mismo sello.

Una de las funciones distintivas de los partidos políticos es la selección de los líderes que competirán por los cargos públicos. Por ejemplo, Dalton y Wattenberg (2000, 5-8) señalan que como los partidos buscan controlar el aparato de gobierno colocando candidatos propios, funcionan como plataforma de selección de líderes; buscan, monitorean y designan candidatos para que compitan por cargos bajo el sello del partido, por lo que sus estructuras internas se emplean para identificar liderazgos y entrenar elites, mediante una carrera partidaria.

Debido a las características del régimen político y la competencia electoral durante los años de gobierno del PAN, es necesario considerar otras funciones y dimensiones de los partidos, más allá de los propósitos electorales. Strom y Müller (1999, 5-13) establecen tres comportamientos partidarios posibles: la búsqueda de cargos, de políticas y de votos. El primero se refiere a los partidos que buscan maxi- 
mizar su control sobre los beneficios o bienes privados, derivados de la designación discrecional en puestos gubernamentales. El segundo alude a maximizar su impacto en las políticas públicas y el tercero a maximizar su apoyo electoral para acceder al control del gobierno. Katz $(2002,94)$ afirma que la maximización de beneficios o recompensa personal derivada del cargo no sólo implica el beneficio material/salarial, legal o ilegal, sino también la recompensa psicológica derivada del estatus y el poder del cargo.

Si bien los propósitos electorales pueden ser predominantes, Epstein (1967, 98-99) sostiene que en el análisis no se deben omitir los casos en los que los líderes partidarios crean estructuras no electorales. Los ejemplos más obvios, señala, son los partidos comunistas y fascistas, cuyos miembros son reclutados para cumplir objetivos mucho más demandantes que los electorales (cuasi militares, revolucionarios); muchos de sus aparatos organizacionales se crearon y mantuvieron con propósitos no electorales, ya que la captura de votos no siempre era necesaria. Epstein sostiene algo similar acerca de los partidos democráticos; los que comenzaron como movimientos de trabajadores en el siglo XIX en Europa no buscaban votos, sino que defendían una causa mediante la propaganda, la presión económica y la organización de los sindicatos; sólo se trasformaron en máquinas electorales de forma secundaria, o después se volvieron electoralistas. Así, Epstein no entiende que los partidos hayan evolucionado de los de notables a los de masas -como lo hace Duverger-, sino que afirma que los partidos modernos de masas son respuestas de movimientos que al principio estaban comprometidos con tareas distintas a las electorales y, por lo tanto, son agrupaciones diferentes a las de los partidos electoralistas. Por eso dice que es necesario mirar con escepticismo la hipótesis de la imitación ("contagio") por parte de los partidos no socialistas a las asociaciones de masas laboristas o socialistas; estas últimas son un tipo especial, situadas en un tiempo y espacio específico, más que una forma típica de las naciones democráticas avanzadas. Epstein señala que el tipo de organización que se creará y mantendrá es en función de la utilidad respecto de los propósitos partidarios; sólo si los líderes desean ganar votos y elecciones conformarán organismos electoralistas (Epstein 1967, 100-102). La idea de que los resultados pueden ser función de utilidad de los líderes es, de 
algún modo, una reflexión que comparte Aldrich $(1995,5)$, al afirmar que los partidos son "instituciones endógenas" a los líderes políticos. Y también señala, sin dejar de considerar las elecciones, que los objetivos de los políticos -éxito y duración en su cargo, poder y prestigio- se alcanzan en el gobierno y no en los partidos, pero que, no obstante, a veces se pueden conseguir mediante ellos; son las acciones de los líderes políticos las que tienen como resultado a los partidos. Los votantes no desempeñan cargos ni buscan beneficios, por lo que no son integrantes de los partidos, aunque se identifiquen con ellos y los apoyen enérgicamente (Aldrich 1995, 20-21). Entonces, Aldrich se pregunta ¿cuándo deciden los líderes crear, modificar o usar a los partidos?, "la respuesta es que los partidos son diseñados como intentos para resolver problemas que los arreglos institucionales vigentes o predominantes no resuelven, y que los políticos creen que no podrán resolver" $(1995,22)$.

Es interesante mencionar algunas teorías sobre los partidos, que se focalizan en las condiciones que regulan la competencia política ya que, como se indicó, los propósitos de los líderes en cada contexto y régimen político repercuten en los tipos de organización resultante. Por esta razón, las definiciones de partidos deben también considerar -como proponía Epstein- los entornos no competitivos en los que actúan. Por ejemplo, Lipset y Rokkan $(1992,235)$ afirman que en los sistemas monolíticos los ciudadanos no diferencian entre el sistema político y los funcionarios que ocupan los cargos, sino que identifican la organización política con la política de dirigentes particulares, que explotan lealtades nacionales para obtener apoyos personales. ${ }^{10} \mathrm{La}$ categoría de sistema monolítico refiere a una complejidad social baja. LaPalombara y Weiner (1966, 3-6) señalan que los partidos políticos surgen cuando la actividad de un sistema político alcanza cierto grado de complejidad, o cuando la noción de poder político considera la idea de que las masas deben ser controladas o incorporadas. Aquí es

10 Además, los autores señalan que estos sistemas no producen oposiciones funcionales, ya que éstas sólo se pueden desarrollar después de la consolidación del territorio nacional. En efecto, en un primer momento, la conformación de la burocracia nacional puede producir oposiciones territoriales que conducen a la guerra o la secesión, pero una vez consolidado el ámbito de actividad de la burocracia gubernamental se pueden producir alineamientos más complejos (Lipset y Rokkan 1992, 244-245). 
interesante la cuestión del control de las masas, ya que da cuenta de los partidos que surgen en sistemas no competitivos (totalitarios o autoritarios).

Tipo de partido: relación partidaria con el Estado y con los ciclos institucionales

Este punto puede ser útil para establecer si el PAN fue una "fiel versión del partido europeo de notables" (Malamud 2009, 343), o si es posible encontrar algunas diferencias. LaPalombara y Weiner $(1966,7)$ afirman que "los pequeños grupos oligárquicos", que se autodenominaron partidos en América Latina, "son más parecidos a las facciones de notables de la República Romana [...] que a partidos políticos preocupados por ganar o mantener el apoyo popular propios de las democracias modernas o de los Estados totalitarios". Como ya se dijo, si bien es posible observar cierta preocupación de las elites del PAN por obtener apoyos populares, éstos sólo funcionaban como legitimación posterior de las autoridades designadas o impuestas de forma coactiva, de modo que la apreciación de LaPalombara y Weiner sobre las oligarquías latinoamericanas parecería apropiada. No obstante, como se vio en el primer apartado, el desarrollo y la utilización del Estado como recurso para la obtención de lealtad indica algunas diferencias con los partidos de notables.

Para estudiar los tipos de partidos políticos que aparecieron en momentos históricos distintos, Katz y Mair $(2004,10)$ dejan de lado la concepción evolutiva de sus organizaciones, y proponen estadios sucesivos en los que la acción de los partidos genera reacciones que, a su vez, producen otros nuevos en un proceso continuo. El primero de los estadios es el de los regímenes censitarios de fines del siglo XIX y principios del xx, organizado con base en el sufragio restrictivo frente a sectores no propietarios de la sociedad. En éste, los autores sostienen que la división entre Estado y sociedad era débil, como en los sistemas monolíticos mencionados. La población que ocupaba todos los puestos en el Estado estaba muy ligada o era equivalente a la que se posicionaba en los segmentos superiores de la sociedad; Estado y sociedad estaban interconectados, y los partidos de notables se encontraban en su intersección: “los partidos eran básicamente comités formados 
por aquellos que conjuntamente constituían tanto el Estado como la sociedad civil" (Katz y Mair 2004, 16). Duverger (1980) consideraba que en el siglo xx este tipo de partidos sólo subsistía en Estados Unidos (encarnado por el caucus system: aparatos privados e intermitentes de movilización electoral); su conceptualización del "partido de cuadros" lo ubicaba en un continuo antiguo/moderno, que correspondía a los partidos de notables de creación electoral-parlamentaria o de origen interno al ciclo institucional, y que a raíz de las amenazas del entorno (sufragio ampliado, partidos de creación externa -cooperativas agrícolas y sindicatos-) se trasformarían indefectiblemente en partidos de masas, y así completarían su supuesto "ciclo evolutivo". El estadio de los "partidos cartel” implicaría, de algún modo, un retorno al Estado, luego de una posición intermedia durante los estadios del partido de masas y del catch-all; éste tiene lugar a partir del declive de la participación e implicación ciudadanas en la actividad partidaria. Así, los partidos se han visto obligados a buscar recursos en otra parte: subvenciones estatales (que permiten su permanencia en el poder y al mismo tiempo impiden el acceso de grupos nuevos) y control de medios audiovisuales. Así, el Estado, "invadido" por los partidos se vuelve una fuente de recursos mediante la cual los del tipo cartel aseguran su supervivencia y refuerzan su capacidad de resistencia ante los outsiders:

El Estado se convierte en una estructura institucionalizada de apoyo, respaldando a los insiders y excluyendo a los outsiders. Los partidos pasan a ser absorbidos por el Estado [...] Habiendo anteriormente asumido el papel de tutores, más tarde de delegados, y después, en el apogeo del partido catch-all, de empresarios, los partidos se han convertido en agencias semi-estatales (Katz y Mair 2004, 25).

Existen otros análisis acerca de la relación entre Estado y partidos, que muestran trayectorias distintas a la secuencia planteada por Katz y Mair, cuando se observa a los partidos dependientes del Estado anteriores al estadio del cartel. Puhle (2007, 85-86) analiza el Parteinstaat (que podría traducirse como "Estado de partidos"), desarrollado primero en las democracias posfascistas de la década de 1950 (República Federal de Alemania, Austria e Italia) y luego, durante los años setenta, en Grecia o España. Parteinstaat o Partitocrazia "es una situación 
en la que los partidos políticos dominan el Estado y 'colonizan' importantes segmentos de sus instituciones y de la sociedad, como la Administración Pública (a todos sus niveles), las empresas públicas, la educación, los medios de comunicación, etc.”. De manera similar a lo propuesto por Katz y Mair, Puhle señala que tanto los partidos de gobierno como los de oposición explotan los privilegios y servicios del Parteinstaat, "a pesar de su competición electoral, los partidos conforman en muchos sectores un cartel oligopólico que dirige importantes instituciones y recursos estatales" $(2007,86)$.

Homogeneización estatal y faccionalización partidaria: incentivos, competencia e institucionalización de los partidos políticos

Huntington (1996) dice que los partidos políticos atraviesan fases distintas antes de que su organización culmine en la institucionalización. Un partido institucionalizado permite incorporar grupos a la participación política, sin que ello altere la estabilidad del sistema político. La primera fase es la del faccionalismo: la participación y la institucionalización son bajas, y si bien los grupos e individuos manifiestan actitudes no tradicionales, aún no se han desarrollado organizaciones modernas. En esta fase

la política abarca a muy pocas personas que compiten entre sí, en medio de muchas alianzas y agrupamientos débiles y transitorios. Los agrupamientos tienen escasa durabilidad y ninguna estructura. En general son las proyecciones de ambiciones individuales en el contexto de rivalidades y afiliaciones personales y de familia. Estos grupos políticos pueden ser denominados partidos, pero carecen de la organización continuada y del apoyo social que son la esencia de un partido (Huntington 1996, 362). ${ }^{11}$

11 Kitschelt $(2000,849)$ también analiza la relación entre ambición individual y estructura partidaria, y afirma que cuando los políticos no enfrentan desafíos electorales lo que mantiene unificada a la organización es la autoridad carismática del liderazgo. Cuando los políticos invierten sólo en técnicas de resolución de problemas, pero no en infraestructura organizacional, construyen caucus legislativos o facciones, y es lo que sucede en oligarquías competitivas con sufragio restringido. 
Si el sistema político cuenta con legislaturas, las facciones se orientarán a controlarlas para establecer relaciones interelitistas, y no se relacionarán con las masas. Por otra parte, los candidatos se seleccionan como individuos y sobre la base de su posición económica (Huntington 1996, 363). Cuando no hay legislaturas ni elecciones, la forma dominante de facción prepartidaria es la conspiración revolucionaria. Así, la competencia entre facciones funciona como un sistema cerrado en el que sus miembros modifican continuamente a sus socios y antagonistas sin aumentar el número de participantes (Huntington 1996, 364).

Boucek $(2009,455)$ describe tres tipos de faccionalismo partidario: el cooperativo, el competitivo y el degenerativo. El primero ocurre en partidos cuya estructura facciosa tiene el potencial de incrementar la capacidad agregativa y de facilitar la cooperación entre ellos, cuando existen incentivos centrípetos. Así proveen una estructura de cooperación entre los grupos; el faccionalismo puede diversificar las adhesiones y acelerar la integración partidaria. El segundo se puede presentar después de la etapa formativa del partido, y suele estar asociado a la competencia centrífuga que desatan los desacuerdos internos; también implica fragmentación y rupturas y, si bien la competencia entre facciones no es necesariamente perjudicial, puede ser difícil de administrar (Boucek 2009, 469-474).

El faccionalismo degenerativo puede destruir al partido; si las facciones adquieren poder y crecen sin control puede haber procesos degenerativos de fragmentación. Existen tres subtipos de faccionalismo degenerativo: a) el de fragmentación excesiva que, junto con la difusión del poder, complica la formación de mayorías y puede trasformar a las facciones en actores de veto; b) el de incentivos "privatizantes", que emerge si hay una división de los beneficios materiales entre cada facción, ya que el comportamiento "autointeresado" conduce a que la atención en los propósitos partidarios quede marginada en pos de los intereses inmediatos de los líderes de cada facción; el sistema de facciones puede pasar a ser sólo una red compleja de relaciones clientelares y un reservorio de bienes privados que produzcan la pérdida de adhesiones y el despilfarro de recursos y c) el enraizado, que es cuando la búsqueda personalizada de votos juega un rol importante en el enraizamiento de las facciones en plano territorial, pues las empuja a descentralizar sus actividades, mientras que el proceso de 
obtención de votos crea una miríada de redes verticales de patronazgo político en las comunidades locales, que multiplica las oportunidades para el desvío de recursos públicos. La división extrema de los bienes (spoils) es insostenible en el largo plazo, porque los recursos estatales son limitados; además, la captura del partido por parte de una facción puede activar un ciclo destructivo de faccionalización, y los partidos que monopolizan el poder por un largo periodo están muy expuestos al riesgo de captura (Boucek 2009, 477-478).

Panebianco (1993, 215-217) estudió también los procesos de institucionalización y faccionalización, y propuso una división interesante entre los partidos que se institucionalizan como gobernantes, y aquéllos que atraviesan esta etapa por fuera del Estado o en la oposición. Es posible observar partidos que llevaron a cabo su institucionalización desde una situación "central”, que accedieron al gobierno nacional tras su nacimiento, antes de que se consolidaran sus organizaciones, y que permanecieron en el poder durante largos periodos. Durante la consolidación de los partidos de gobierno, el control que éste ejerce favorece una institucionalización débil, ya que disponer de los recursos públicos constituye un factor inhibitorio para que el desarrollo de su organización sea fuerte. La cantidad de recursos públicos utilizados para fines "privados" (para la lucha política) afecta el proceso de institucionalización de los partidos: a más recursos disponibles, menos necesidad de promover una fuerte institucionalización. Además, también influyen otros dos factores: la burocracia estatal y la competitividad del sistema político. Si la burocracia permite una disponibilidad máxima de recursos públicos, plausibles de ser redistribuidos en forma de incentivos organizativos (spoils system), la institucionalización de los partidos será débil. La disponibilidad amplia de recursos no motiva a los líderes a crear burocracias partidarias fuertes, ya que los incentivos selectivos ${ }^{12}$ son gubernamentales. Por el contrario, si la burocracia es poco susceptible de ser colonizada, por lo que hay menos recursos públicos disponibles, los partidos serán más institucionalizados; al no existir recursos alternativos, los líde-

12 Según Panebianco, los incentivos selectivos son recursos que se intercambian entre líderes (en una relación de poder horizontal) o entre líderes y seguidores (en una relación de poder vertical), con miras a mantener la coalición dominante de una agrupación política y su supervivencia, en tanto que organización (1993, 64-69). 
res deben desarrollar la organización del partido. Por otra parte, la intervención del Estado en la economía cumple la misma función en cuanto a la posibilidad de "privatizar" recursos (Panebianco 1993, 217). La competitividad del sistema político también repercute en la institucionalización de los partidos, ya que si éstos no enfrentan amenazas reales a su condición de partido gobernante no desarrollarán las organizaciones necesarias para la competencia política. Cuando no existe incertidumbre, hay menos incentivos para crear burocracias internas estables, para la creación de áreas encargadas de la comunicación con el entorno.

\section{Análisis teórico del PAN: un partido estatal de facciones}

Si se aplican las definiciones acerca de los partidos y los análisis sobre la competencia política, el acceso al poder y el reclutamiento de liderazgos, contemplando la reconstrucción histórica del primer apartado, es posible afirmar que las definiciones que consideran que los partidos cumplen la función de estructuración legal del voto y de selección de los líderes que ocuparán cargos públicos no se pueden aplicar al caso del PAN porque, en efecto, éste carecía de mecanismos legales estables -formales o informales- para seleccionar liderazgos. El proceso de selección y disputa entre facciones del PAN se resolvía mediante las intervenciones federales, las revoluciones y la utilización del régimen político (la "unificación del origen electoral" y la designación presidencial de los cargos ejecutivos, legislativos y de los electores). Por otra parte, el pool de líderes disponibles estaba determinado en primer lugar por la posición económico-estructural personal en cada provincia y, en segundo, por la manifestación de apoyo a la facción predominante, por lo general, a la presidencial, que se basaba en intercambios materiales. En este sentido, el PAN no era la estructura de la cual emergían los líderes, sino que estos pre existían y eran seleccionados por un sistema asociado al régimen, es decir, designaciones e intervenciones. No obstante, si bien las elecciones no determinaban quién accedía a los cargos, sí funcionaron como un método de legitimación ex post de la resolución intraelitista de la disputa entre facciones. Por esta razón, el sistema de producción de votos 
y de movilización electoral -conformado por uno que combinaba presencia militar, estatus personal, jueces de paz y caudillos locales-sí permite afirmar que el PAN contó con organizaciones especiales para la movilización política con miras al control de las masas. Esto muestra el interés de las elites del PAN por obtener apoyos populares para mantener un poder cuasi de facto.

Si bien la concurrencia al partido como plataforma para el acceso al poder no implicaba un proceso de selección y competencia, sí manifestaba la función de utilidad para los líderes de cada facción local o provincial, ya que cada uno ingresaba al PAN sólo en los momentos previos a cada elección, lo que permite observar a sus facciones como instituciones endógenas a los líderes territoriales. En el primer apartado se describió el modo en que se materializaba la retribución de los apoyos a las facciones, por ejemplo, en recompensas mediante cargos o tierras, por lo que es posible sostener que el PAN se caracterizó por ser un partido orientado a los cargos y maximizador de los beneficios personales de los líderes territoriales. Por otra parte, si el régimen muestra características monolíticas de equivalencia tanto entre los ocupantes de la cima de la pirámide económica como los de la política, y el contexto muestra una miríada de conflictos u oposiciones territoriales -desatadas por la expansión de la burocracia central/nacional-, también es evidente que los alineamientos políticos hayan manifestado poca oposición funcional. En cuanto a esto último, la relación clase/voto en los centros urbanos no afectó la competencia entre las elites ya que, aunque haya evidencia de electorados distintos para cada partido, no se observa una disputa por su captura o representación.

Por otra parte, a partir de la relación del PAN con el Estado y con los ciclos institucionales es posible determinar si se puede categorizar como un partido de notables. Según la reconstrucción histórica realizada en el primer apartado, se podría sostener que el PAN no constituyó íntegramente un partido de notables. Si sólo se consideran las características, de algún modo, estáticas del "régimen oligárquico" o del "bloque conservador", es probable que se llegue a concluir que el PAN sí fue un partido de notables. Lo que habilitaría esta conclusión es la equivalencia estructural entre los poseedores de tierras y las elites dirigentes, el sistema de designaciones presidenciales de cargos, la dis- 
tribución intraelitista de privilegios, la restricción de la participación política y la intermitencia de las maquinarias electorales, en tanto que única estructura partidaria. Si se considera el ciclo institucional y los usos del Estado, se podría matizar la afirmación de que el pan fue un partido de notables. En primer lugar, los ciclos institucionales y el sistema de acceso y distribución de cargos (el régimen) materializaban los resultados de la resolución de conflictos facciosos que ocurrían por fuera del ciclo institucional. Si bien no es posible afirmar que el PAN fue un partido de "creación externa", de acuerdo con Duverger, tampoco fue uno que emplease del todo las instituciones representativas de gobierno, como las parlamentarias, como en el caso europeo, para distribuir privilegios y dirimir conflictos políticamente. De hecho, las instituciones empleadas fueron los instrumentos coactivos formales de los poderes ejecutivos nacionales, provinciales y locales -para intervenir provincias y redistribuir recursos materiales-, y los instrumentos informales asociados tanto a los caudillos locales como a los recursos provistos por la ocupación de la administración pública (levantamientos, financiamiento de revoluciones); además, la presencia militar en las provincias era un componente crucial de la base de poder y control local de cada facción.

En cuanto a los usos del Estado y a la posición del partido respecto de éste y la sociedad, se debe señalar que hay una diferencia entre la presencia u ocupación del Estado por parte de los segmentos superiores de la sociedad, y el empleo de los recursos estatales para el armado de coaliciones políticas. Si la utilización de recursos públicos para activar las maquinarias electorales o para recompensar apoyos, mediante el empleo público, es una característica compartida por el PAN y otros partidos notables, es posible afirmar que el PAN también comparte algunos rasgos del "Estado de partidos" y de los partidos cartel, al menos en cuanto a usos modernos de los recursos estatales. El PAN funcionó como un sistema de partidos en sí mismo, que expulsaba a las facciones que no demostraban lealtad; al existir una homología estructural entre el sistema político y el PAN, no había posibilidad de ingresar al Estado más que mediante la violencia. El Estado, entonces, manifestaba características cartelizadas u oligopólicas en cuanto a la competencia, que no se observan de forma tan clara en los procesos más competitivos protagonizados por los partidos de notables de las 
asambleas constituyentes francesas de 1789 y 1848, del Parlamento de Francfort de la misma época, de los grupos parlamentarios surgidos en Suecia y Suiza luego de la introducción del sistema proporcional, o de la Italia del sistema Giolitti (Duverger 1980, 16-19).

Lo interesante en este aspecto es que si bien las teorías sobre "Estados de partidos" y "cartelización” señalan que estas características se deben a condiciones históricas específicas, asociadas a la supervivencia de las organizaciones en coyunturas de debilitamiento de sus vínculos con la sociedad, o como consecuencia del surgimiento de clivajes nuevos, a partir de la reconstrucción histórica realizada es posible observar que el empleo y la dependencia de los recursos estatales para la supervivencia política de las organizaciones partidarias podría encontrarse en periodos históricos previos a los señalados por los estudios mencionados. En este sentido, se reitera lo que en este trabajo se entiende por uso coalicional de "recursos estatales", según lo observado en la reconstrucción histórica: privatizaciones y nacionalizaciones de tierras, cargos en todos los niveles de la administración pública y el gabinete ejecutivo nacional, creación de empresas y entes públicos -ferrocarriles y bancos- y discrecionalidad tarifaria, crediticia y monetaria en la administración de esas agencias, distribución selectiva de obras públicas y de puestos de empleo derivados de ellas, subsidios a las provincias receptoras de "colonias oficiales", intervenciones federales, mediante el uso del Ejército, y movilizaciones electorales a través de jueces de paz y del patronazgo. Estos recursos estatales, como se sostiene en este artículo, fueron empleados para obtener la lealtad y adhesión de las facciones territoriales del PAN, lo que repercutió en dos dinámicas interconectadas; al tiempo que se desarrollaron instituciones de alcance nacional, el proceso de institucionalización del PAN fue débil.

En relación con los incentivos estatales, la institucionalización partidaria y la competencia política, es posible sostener que la posición central del PAN en tanto que partido de gobierno influyó en su proceso de institucionalización, lo que resultó en un partido faccionalizado. Al mismo tiempo, esta baja cohesión manifestó las características de un faccionalismo degenerativo, ya que es posible encontrar incentivos "privatizantes", facciones enraizadas y fragmentación excesiva. Entre 1874 -administración de Avellaneda-y 1916 -primera de Yrigoyen- 
una facción del PAN ocupó la presidencia de la nación: durante 42 años el PAN fue un partido de gobierno (si sólo se contabilizan las presidencias, es decir, al margen de los gobiernos provinciales a los que pudo acceder una facción del PAN). Pero, al mismo tiempo, sufrió más de diez rupturas y, durante los años que gobernó existieron 23 agrupamientos que respondían a liderazgos territoriales distintos. La explicación que se ofrece aquí es que el PAN fue un partido de gobierno, que no solo tuvo los recursos estatales disponibles para cualquier actor gubernamental, sino que además fue el encargado de desarrollar un amplísimo conjunto de instituciones estatales nuevas de alcance nacional.

De acuerdo con Panebianco (1993), estos recursos se pueden considerar como incentivos selectivos, gubernamentales o estatales, que inhibieron el desarrollo de burocracias partidarias o bien, según Boucek (2009), como incentivos "privatizantes" que trasformaron al PAN en una simple red de beneficios materiales y de redistribución entre facciones de bienes privados. Al mismo tiempo, en el modo en que las ligas provinciales dirimían sus conflictos y movilizaban a la población durante las elecciones, es posible observar un tipo de faccionalismo enraizado en el que la obtención de votos activaba prácticas de patronazgo y desvío de recursos. Finalmente, otros dos factores que quizá determinaron que el PAN fuera un partido de facciones son la posibilidad de colonizar las burocracias -prácticamente el PAN creó las nacionales y provinciales- y la baja competencia política abierta, -ya que no fue necesario "ganar" electores sino sólo movilizarlos, pues los conflictos se resolvieron mediante intervenciones federales y revoluciones-, lo que volvió innecesaria la existencia de sectores de la organización partidaria o burocracias preparadas para la competencia electoral.

\section{Comentarios finales}

Aquí se definió al PAN como un partido estatal de facciones, en un intento por captar tanto su dependencia organizacional respecto de los recursos estatales, empleados para producir la adhesión de las subunidades partidarias, como los efectos del uso de estos recursos sobre su 
institucionalización y sobre la creación de órganos estatales de alcance nacional. Por lo tanto, este trabajo podría contribuir no sólo a las discusiones históricas del periodo analizado aquí, sino también a algunas sobre las trasformaciones organizacionales de los partidos políticos, tanto sobre procesos de formación estatal como de politización de las burocracias. En relación con dichas trasformaciones, el análisis del PAN permite pensar que la supervivencia de los partidos no pasaría a depender del Estado sólo cuando se agotan otras fuentes de recursos (Katz y Mair 2004; Puhle 2007), sino que el uso y la necesidad de fondos estatales podría estar asociado con la búsqueda de adhesión política en contextos en los que otras fuentes de recursos aún no se encontraban activas; la trasformación de los partidos en "agencias semiestatales" no sería, en el caso del PAN, un estadio posterior al colapso de las identidades partidarias, a la ampliación de los electorados o al declive del interés ciudadano en la participación política sino que, por el contrario, sería un estadio inicial o incluso permanente. Sería interesante analizar si se ha modificado esta dependencia de los recursos estatales durante etapas posteriores a la observada aquí, aunque existe cierta evidencia de que, como en el caso de la Unión Cívica Radical mencionado antes, esa dependencia fue más bien permanente (Horowitz 2007; Rock 1972; Walter 1974; Calvo y Murillo 2005; Levitsky 2005; Scherlis 2013)

Es precisamente a partir de este atributo estatista del pan que aquí se ha cuestionado la pertinencia de la categoría de "partido de notables”, para describirlo. Por otra parte, el análisis del PAN, en relación con el proceso de formación del Estado argentino, podría contribuir también a revelar una dinámica no siempre considerada por el campo de estudios sobre construcción estatal. Por ejemplo, los trabajos de Oszlak (2009; 1982) sobre el Estado argentino sostienen que éste habría sido producto de la necesidad de las elites agroexportadoras de autoproveerse de una estructura institucional capaz de garantizar y reproducir su actividad y dominación. Algunos estudios sobre otros casos consideran que el Estado sería resultado de la necesidad gubernamental de extraer recursos de la sociedad para el financiamiento de guerras (Tilly 1975) o la distribución de bienes públicos (Levi 1988). Otros análisis consideran que el Estado resultaría de la necesidad de emprender obras públicas de gran escala (Wittfogel 1957), mien- 
tras que algunos otros se enfocan en el entorno ecológico (Carneiro 1970), o en el control territorial (Mann 2008).

El análisis del PAN, propuesto aquí, permitiría complementar estas discusiones: las instituciones estatales podrían ser resultado de la competencia entre facciones partidarias que emplearían recursos públicos para obtener lealtad y adhesión política; como se intentó mostrar en la reconstrucción del periodo analizado, las remuneraciones coalicionales habrían conducido a la expansión y creación de entes estatales de alcance nacional. Y, en relación con esto último, el trabajo podría también complementar algunas discusiones acerca de la politización de las burocracias y la autonomía o captura del aparato estatal por parte de partidos y liderazgos políticos (Zuvanic y Iacoviello 2010; Evans 1995; Peters y Pierre 2004, entre otros). En el caso revisado aquí, se pueden observar algunos ejemplos interesantes que muestran que el aparato estatal -e incluso las empresas públicas- no se encontraría aislado de la competencia política ni sería un ámbito exterior a ella sino que, por el contrario, la política -en tanto que intercambio de recursos estatales por lealtad-, se encontraría en el origen mismo de las burocracias.

\section{Bibliografía}

Adamovsky, Ezequiel. 2009. Historia de la clase media argentina: apogeo y decadencia de una ilusión, 1919-2003. Buenos Aires: Planeta.

Aldrich, John. 1995. Why parties?: the origin and transformation of political parties in America. Chicago: The University of Chicago.

Alonso, Paula. 2009. El Partido Autonomista Nacional y las elites políticas en la Argentina de fin del siglo XIX. Anuario IHES (24): 369-388.

Alonso, Paula. 2003. La política y sus laberintos. El Partido Autonomista Nacional entre 1880 y 1886. En La vida política. Armas, votos y voces en la Argentina del siglo xix, coordinado por H. Sabato y A. Lettieri, 277-292. Buenos Aires: Fondo de Cultura Económica. 
Botana, Natalio. 2012. El orden conservador. La política argentina entre 1880 y 1916. Buenos Aires: Edhasa.

Boucek, Francoise. 2009. Rethinking factionalism: typologies, intraparty dynamics and three faces of factionalism. Party Politics 15 (4): 455-485.

Bragoni, Beatriz y Eduardo Míguez. 2010. De la periferia al centro: la formación de un sistema político nacional, 1852-1880. En Un nuevo orden político:provincias y Estado nacional, 1852-1880, coordinado por B. Bragoni y E. Míguez, 9-28. Buenos Aires: Biblos.

Calvo, Ernesto y María Victoria Murillo. 2005. A new iron law of Argentine politics? Partisanship, clientelism, and governability in contemporary Argentina. En Argentine democracy: the politics of institutional weakness, editado por S. Levitsky y M.V. Murillo, 207-226. Pensilvania: The Pennsylvania State University.

Cantón, Darío y Jorge Jorrat. 2004. Clase social y voto en la ciudad de Buenos Aires: 1864-1910. Revista SAAP 2 (1): 11-33.

Carneiro, Robert. 1970. A theory of the origin of the state. Science, New Series 169 (3947): 733-738.

Dalton, Russell y Martin Wattenberg. 2000. Unthinkable democracy. Political change in advanced industrial democracies. En Parties without partisans: political change in advanced industrial democracies, editado por R. Dalton y M. Wattenberg, 3-16. Nueva York: Oxford University Press.

Downs, Anthony. 1957. An economic theory of democracy. Nueva York: Harper.

Duverger, Maurice. 1980. Partidos políticos. México: Fondo de Cultura Económica.

Epstein, Leon. 1967. Political parties in western democracies. Nueva York: Praeger. 
Evans, Peter. 1995. Embedded autonomy: states and industrial transformation. Nueva Jersey: Princeton University Press.

González, Carlos Aurelio. 2000. Breve historia del presupuesto en la Argentina. Revista ASAP 35: 119-166.

Herrera, Claudia. 2010. Fiscalidad y poder: las relaciones entre el estado tucumano y el Estado central en la formación del sistema político nacional, 1852-1869. En Un nuevo orden político: provincias y Estado nacional, 1852-1880, coordinado por B. Bragoni y E. Míguez, 181-207. Buenos Aires: Biblos.

Hora, Roy. 2001. Autonomistas, radicales y mitristas: el orden oligárquico en la provincia de Buenos Aires (1880-1912). Boletín Ravignani III (23): 39-77.

Horowitz, Joel. 2007. Patrones y clientes: el empleo municipal en el Buenos Aires de los primeros gobiernos radicales (1916-1930). Desarrollo Económico 46 (184): 569-596.

Huntington, Samuel. 1996. El orden político en las sociedades en cambio. Barcelona: Paidós.

Ignazi, Piero. 1996. The decline of parties and the rise of new political parties. Party Politics 2 (4): 549-566.

Katz, Richard. 2002. The internal life of parties. En Political parties in the new Europe, editado por K. Luther y F. Müller-Rommell, 87-118. Nueva York: Oxford University Press.

Katz, Richard y Peter Mair. 2004. El partido cartel. La transformación de los modelos de partidos y de la democracia de partidos. Zona Abierta 108-109: 9-42.

Kitschelt, Herbert. 2000. Linkage between citizens and politicians in democratic polities. Comparative Political Studies 33 (6/7): 845-879. 
LaPalombara, Joseph y Myron Weiner. 1966. The origin and development of political parties. En Political parties and political development, editado por J. LaPalombara y M. Weiner, 3-42. Princeton: Princeton University Press.

Lettieri, Alberto. 1999. Repensar la política facciosa: la conciliación de los partidos políticos de 1877 en Buenos Aires. Boletín Ravignani III (19): 35-80.

Levi, Margaret. 1988. Of rule and revenue. Berkeley: University of California Press.

Levitsky, Steven. 2005. La transformación del justicialismo. Del partido sindical al partido clientelista, 1983-1999. Buenos Aires: Siglo XXI Editora Iberoamericana.

Lipset, Seymour y Stein Rokkan. 1992. Estructuras de división, sistemas de partidos y alineamientos electorales. En Diez textos básicos de ciencia política, AAVv, 231-273. Barcelona: Ariel.

Malamud, Andrés. 2009. Partidos políticos. En Introducción a la ciencia política, compilado por J. Pinto, 321-350. Buenos Aires: EUDEBA.

Mann, Michael. 2008. Infrastructural power revisited. Studies in Comparative International Development 43: 355-365.

Oszlak, Oscar. 2009. La formación del Estado argentino. Buenos Aires: Emecé Editores.

Oszlak, Oscar. 1982. Reflexiones sobre la formación del Estado y la construcción de la sociedad argentina. Desarrollo Económico XXI (enero-marzo): 1-18.

Panebianco, Angelo. 1993. Modelos de partido. México: Alianza.

Paz, Gustavo L. 2010. Años turbulentos. Política provincial e instituciones nacionales: Jujuy, 1875-1880. En Un nuevo orden político: pro- 
vincias y Estado nacional, 1852-1880, coordinado por B. Bragoni y E. Míguez, 159-179. Buenos Aires: Biblos.

Peters, Guy y Jon Pierre. 2004. Politicization of the civil service in comparative perspective. The quest for control. Londres: Routledge.

Puhle, Hans Jürgen. 2007. Crisis y cambio de los partidos catch-all. En Partidos políticos.Viejos conceptos y nuevos retos, editado por J. Montero, R. Gunther y J. Linz, 71-100. Madrid: Editorial Trotta.

Rock, David. 1972. Machine politics in Buenos Aires and the Argentine radical party, 1912-1930. Journal of Latin American Studies 4 (2): 233-256.

Rock, David y Fernando López-Alves. 2000. State-building and political systems in nineteenth-century Argentina and Uruguay. Past \& Present 16: 176-202.

Sánchez Román, José Antonio. 2010. Integración territorial y especialización económica. Tucumán y el Estado nacional, 1850-1880. En Un nuevo orden político: provincias y Estado nacional, 1852-1880, coordinado por B. Bragoni y E. Míguez, 209-225. Buenos Aires: Biblos.

Scherlis, Gerardo. 2013. The contours of party patronage in Argentina. Latin American Research Review 48 (3): 63-84.

Schlesinger, Joseph. 1991. Political parties and the winning of office. Ann Arbor: University of Michigan Press.

Strom, Kaare y Wolfgang Müller. 1999. Political parties and hard choices. En Policy, office, or votes?: how political parties in western Europe make hard decisions, editado por W. Muller y K. Strom, 1-36. Nueva York: Cambridge University Press.

Ternavasio, Marcela. 2009. Historia de la Argentina 1806-1852. Buenos Aires: Siglo XXI. 
Tilly, Charles. 1975. The formation of national states in western Europe. Nueva Jersey: Princeton University Press.

Walter, Richard. 1974. Municipal politics and government in Buenos Aires, 1918-1930. Journal of Interamerican Studies andWorld Affairs 16 (2): 173-197.

Wittfogel, Karl. 1957. Oriental despotism. A comparative study of total power. New Haven y Londres: Yale University Press.

Yanai, Nathan. 1999. Why do political parties survive? An analytical discussion. Party Politics 5 (1): 5-17.

Zuvanic, Laura y Mercedes Iacoviello. 2010. La burocracia en América Latina. ICAP-Revista Centroamericana de Administración Pública 58/59: 9-41. 
